\title{
Research On Decision Support Of Whole Process For Mega And Complex Projects
}

\author{
Fan Min $^{1, a}$, Song Shijun ${ }^{2, b}$ \\ ${ }^{1}$ School of Economics and Management, Southwest Jiaotong University, china \\ ${ }^{2}$ Office of Labs and Facilities Management, Southwest Jiaotong University, China \\ afanmine@163.com, bswjtulab@home.swjtu.edu.cn
}

Keywords: Mega and Complex, Project Management, Decision Support

\begin{abstract}
Study on decision support features of mega and complex projects, summarizes the unique characteristics of its decision-making process, described the importance of the whole process of decision-making, combined with the latest advances in data mining technology, applies an coupled model(data mining-decision support)for engineering decision.
\end{abstract}

\section{Introduction}

Project management methods and tools have been undertaken for many years, various decision-making methods and tools continue to improve and realized, more and more experience of the project can be used to sum up, learn, however, the project's decision-makers but found that their decisions more and more difficult to correct, a variety of scientific decision-making methods and applications of the theory does not guarantee that the project decision is entirely correct [1].Many decision-makers using a variety of scientific decision-making tools and methods, butstill unable to break away from the decision-making embarrassing mistakes.To be sure, the scientific theory to guide our decision-making is nothing wrong,leading up to the actual project decision making activities of different result occurs because the project decision makers in different modes of behavior are our policymakers behavioral differences lead to different decisions effect.

\section{Decision-Making of Complex Project}

Facing a complex policy-making question solution, we should first understand which unit of (sub-question, element, etc.), whether there is interaction between the various units, that is, you should first understand the structure of the problem, creating the problem structure model. Problem structure model is the product of rational processing of practical problems, and problems related to the basic elements (arguments, concepts) differentiation in varying degrees, and the objective description of the results.

Due to the mega and complex project itself features, makes the argument in its investment decisions, and unlike most small projects investment decision, not to maximize the economic benefits as a destination for investment decisions, but more to take into account social and eco-environmental benefits of play. For example, the construction of a large water conservancy and hydropower projects, not only to take into account the inputs and outputs of the ratio of funds, and the investment once completed, by electricity or shipping the economic benefits, should also consider if the investment in the project, the resulting impact of migration on social stability, as well as in the construction process influence on eco-environment around, and so on [2-3].

Compared with the general decision-making of project or enterprise profitability, decision-making has a unique characteristic of the mega and complex project, mainly reflected in the following aspects:

(1) The manifestation of the will of the Government's strategy: a period of development in a country or region, mega and complex project often become the central or local governments to develop an important part of the long-term development plan of the economic, technological, or military, reflecting the national economic social development strategy will. 
(2) Consensus strength is formidable: the mega and complex project permanent public welfare characteristic caused its decision-making and the implementation has aroused public's widespread interest, moreover, the general public welfare significant project part investment originated from the public tax revenue, the public should have the right to know to the mega and complex project decision-making, therefore, has formed the formidable consensus strength.

(3) The strong dependence of information: mega and complex project decisions is based on the information that is more historical data as well as forecasts for the future of the scientific data, including the conceptual information and rational information. These data generally refers to projects involving the relevant technology and information, which has a non-real-time, and not to the variability. However, these data access is generally secondary decision-making body of experts to complete.

(4) Time is non-sensitive: for the same decision problem, the timing of different, their ends often have a great deal of difference. Sometimes in order to seize market opportunities, decision makers in the information uncertain or insufficient argumentsmake risky decision. However, the choice of mega and complex project on decision time is non-sensitive, some issues are not fully defined, or there was no appropriate measures proposed, generally should not rush to project or organization.

\section{Decision of Whole Process}

As mega and complex projects involving the complexity of the problem, the complexity of policy makers bounded rationality and decision makers, policy-makers face a dynamic and uncertain decision-making environment [4]. So, the process is very complex for mega and complex projects The process is very complex. Specific large complex engineering, and its decision-making process in general is different for a particular large-scale complex projects, its decision-making process has its own unique personality characteristics and stage, but generally mega and complex project decision-making process, there will be a common stage and characteristics. The general decision-making process of mega and complex projects is shown in Fig.1.

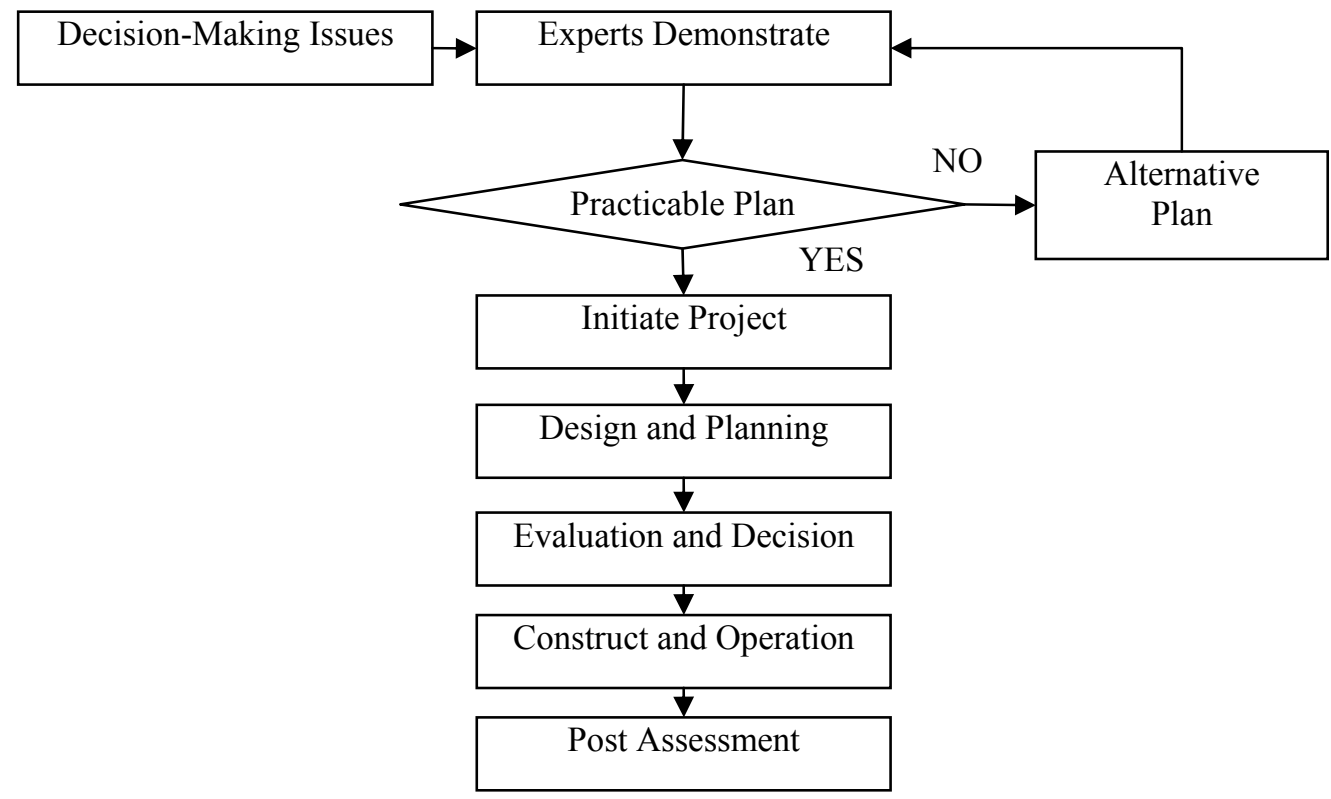

Fig.1 decision-making process for mega and complex projects

Initiate Project. After the project question was proposed, the first policy-making main body(owners) organizes the correlation experts to demonstrate the project. The process of demonstrate feasibility of the project is essential stage. In this stage, we must create certain environmental condition, plays the correlation expert's role fully, guarantees its process and the result is scientific and reasonable. If the conclusion is not feasible, then needs to propose the question temporary processing measure, or proposed the alternative plan, alleviates the influence which the major issue bring. If the plan is feasible, then first policy-making make decision to initiate projects. 
planning and implementation. After Initiated project, planning and design of a project was beginning, and assessment and choice plan. Decision objectives primarily through analysis and comparison of the advantages and disadvantages of the project to finalize the satisfactory plan. Following the selection of the plan, we can construct it and have operation management of the project. during construction of the project, forecasting and decision making for risk of construction are particularly important, senior managers' making the right choice of reasonable prediction and project directly affect the projects' successes and failures. As project complex degree was improved, projects' uncertainty factors gradually increased, this not only including project itself of uncertainty, also including external environment subtle of changes, meanwhile, on project for risk analysis by needed of information quality made has more high of requirements, so, for assist decision-making process in the whole process, if only rely on the Manager's own experience or the team's synergy, it is very difficult to forecasting and make decision.

Post-assessment. Post-assessment is the continuation of mega and complex project decisions, is a modern indispensable phase of mega and complex project throughout the decision-making process. Post-assessment phase of the project, according to the actual running condition and effect, comparison level of deviation between forecast conditions and effect before, exploring the causes of deviations, corresponding measures proposed, raising economic benefit and social benefit projects. For missteps or for other important reasons causing difficulties for the project, post-assessment would offer further decision-making for policy makers to reformulate or select better plan.

\section{Decision Support System Based on Data Mining}

Results of decisions are effected on the decision-makers' professional background, knowledge, experience, character and the impact of a variety of factors such as emotions, with strong subjectivity and uncertainty [5]. For modern projects that increasingly large and complex, and this decision models have been unable to meet the project management in terms of quality, cost, schedule, safety and environmental aspects increasingly demanding requirements. With the development of computer technology, various engineering decision support systems (DSS) are gradually being used to aid engineering decisions. Using DM technology from the engineering data extracted with the knowledge, keep it in a knowledge base of DSS (such as rule database, model database and case database, and so on) and use the appropriate inference mechanism for logic. The key of integration is to solving issues such as knowledge acquisition, expression and use.

Using validation data to test the accuracy of knowledge extraction and calculation accuracy of knowledge based on the test results. In DMDS models, accuracy is an important property of knowledge, plays an important role in decision-making when reasoning. DMDS uses formula (1) to calculate the accuracy of knowledge.

$$
\alpha=\left\{\begin{array}{lr}
\frac{\mathrm{n}^{\prime}}{\mathrm{n}} & \text { Enumeration output } \\
\mathrm{e}^{\frac{-0.7}{\lambda} \frac{\mathrm{v}^{\prime}-\mathrm{v}}{\mathrm{v}}} & \text { Numeric output }
\end{array}\right.
$$

$\alpha$ :Accuracy of knowledge;

$\mathrm{n}$ : The number of records in the validation data

$\mathrm{n}^{\prime}:$ The number of records correctly predicted

$\mathrm{v}$ : Exact value of the predict results

$\mathrm{v}^{\prime}$ : Predictive value

$\lambda$ :Control parameters, expressing the relative error of the forecast result when the accuracy is 0.5

Calculated based on the accuracy of the knowledge used in the process of reasoning predict the accuracy of the reasoning results. Then, coordinating engine uses the following methods to integrate the results from an inference engine.

For numeric element, use reasoning results weighted average values for the final value, such as formula (2) as shown in.

$$
\mathrm{s}=\frac{1}{\mathrm{n}} \sum_{\mathrm{i}=1}^{\mathrm{n}} \alpha_{\mathrm{i}} \mathrm{s}_{\mathrm{i}}
$$


s: final program

$\mathrm{n}$ : the number of reasoning engine

$\mathrm{s}_{\mathrm{i}}, \alpha_{\mathrm{i}}$ : The reasoning results and accuracy of reasoning engine

For enumerated elements, cumulative accuracy of biggest enumeration value is used as the final result. Cumulative accuracy using the enumeration values formula (3) evaluation.

e: One of the enumeration values

$$
\Omega_{\mathrm{e}}=\sum_{\mathrm{i}=1}^{\mathrm{n}} \beta_{\mathrm{i}} \alpha_{\mathrm{i}} \quad \beta_{\mathrm{i}}= \begin{cases}1 & \mathrm{~s}_{\mathrm{i}}=\mathrm{e} \\ 0 & \mathrm{~s}_{\mathrm{i}} \neq \mathrm{e}\end{cases}
$$

$\Omega$ e: The cumulative accuracyof this enumeration value

The DMDS model has the high accuracy and the reliability, can satisfy the project decision-making well the actual operation requirements.

\section{Conclusion}

With the development of computer technology, a variety of decision support system (DSS) is gradually being used to assist in project decision-making. However, subject to knowledge acquisition and data processing obstacles the traditional DSS is difficult to be used widely in engineering practice. On the traditional DSS research and analysis on the basis of the latest developments of data mining technology (data-mining, DM), proposed coupling model for the engineering decisions "data mining - decision support (DMDS).

\section{Acknowledgements}

This work was financially supported by National Key Technology R\&D Program of the Ministry of Science and Technology (NO. 2008BAG07B05).

\section{References}

[1] Shim.J.P, Warkentinm, Courtney.J.F, et al: Decision Support Systems Vol. 33(2002), p. 111-126

[2] Kimgh, Ansh, Kang.K.I: Building and Environment Vol. 39(2004), p. 1235-1242

[3] Bruijn, H.de and A. Porter: Technology Analysis and Strategic Management Vol. 16 (2004), p.261

[4] Smyth, H. J: Journal of Financial Management of Property and Construction Vol. 10 (2005a), p. $33-44$

[5] Darlington, M.J., Culley, S.J.: Advanced Engineering Informatics Vol. 22(2008), p.112-134 